\title{
Material Tests and Mesoscale Computer Model for Ballistic Impact on Carbon Fiber Composite
}

\author{
Sidney Chocron ${ }^{1, *}$, Alexander Carpenter ${ }^{1}$, Rory Bigger ${ }^{1}$, Nikki $\mathrm{Scott}^{1}$, Kyle Warren ${ }^{2}$ and Harun Bayraktar ${ }^{2}$ \\ ${ }^{1}$ Southwest Research Institute, 6220 Culebra Rd., San Antonio, Texas 78238, USA \\ ${ }^{2}$ Albany Engineered Composites, 216 Airport Drive, Rochester, New Hampshire 03867, USA
}

\begin{abstract}
Two-dimensional (unidirectional) and 3-D woven carbon fiber reinforced plastic (CFRP) panels were produced by Albany Engineered Composites. Coupons were machined from the laminates for various mechanical tests in tension, torsion, and delamination. A batch of neat resin was also produced and the mechanical properties of the resin were determined. Some of the mechanical tests were performed at medium and high strain rates. The panels were tested under ballistic impact while recording the back face deflection with a stereo pair of high-speed cameras to perform digital image correlation. Additionally, an ultra-high-speed camera provided a better resolution of the initial $(50 \mu \mathrm{s})$ pyramid that forms after impact. The mechanical tests were used to determine the material properties of the constituents as well as the strength of the interface between matrix and fibers. The properties were incorporated in material models in LS-DYNA to perform simulations of the mechanical tests as well as the ballistic experiments. The ballistic limits, residual velocities, and deflection histories served as a validation of the model and were predicted with good accuracy for two thicknesses of the two-dimensional composite and one of the 3-D composite.
\end{abstract}

\section{Introduction}

The composite material volume in the new Boeing 787 is $80 \%$ by volume or $50 \%$ by weight. This represents a major technology breakthrough as the weight savings is around $20 \%$. The aerospace industry, as well as the automobile industry, use mainly carbon fiber reinforced plastic (CFRP) composites. The reason is that they provide the needed stiffness for the application. But it is well known that CFRPs are not efficient under impact and, in particular, under ballistic impact. This problem is poorly understood and the computer models usually only qualitatively match the ballistic experimental results.

Southwest Research Institute and Albany Engineered Composites teamed to obtain an accurate model capable of predicting ballistic impact perforation and deflection for CFRPs. The technique followed was similar to the one used in the past to generate models for S-2 composites and Kevlar composites. The laminate material was tested in tension, torsion, and delamination. A batch of neat resin was produced and the mechanical properties of the resin were determined. Some of the mechanical tests were performed at medium and high strain rates. The panels were also tested under ballistic impact while recording the back face deflection with a stereo pair of high-speed cameras to perform digital image correlation. An ultra-high-speed camera provided a better resolution of the initial $(50 \mu \mathrm{s})$ pyramid that forms after impact. This paper presents results from part of the tests performed and some of the validation computations. A more detailed paper is expected to be published in the near future.

\section{Materials}

To obtain materials, SwRI partnered with Albany Engineered Composites (AEC), a developer and manufacturer of advanced composite materials used in the aerospace and automotive industries.

\subsection{Materials Description}

AEC delivered two different thicknesses of the 2D unidirectional CFRP material. Panels that were nominally $6.35 \mathrm{~mm}(0.25 \mathrm{in})$ thick were used to machine tensile specimens while panels that were nominally 25.4 $\mathrm{mm}$ ( 1 in) thick were used to machine torsion and delamination specimens. These are described later. Both types of panels were also subjected to ballistic impact tests.

The two-dimensional CFRP composite consists of layers of unidirectional non-crimp fabric (NCF) made from HexTow ${ }^{\text {TM }}$ IM7 PAN-based carbon fibers [1] surrounded by $\mathrm{CYCOM}^{\circledR}$ PR 520 RTM epoxy resin matrix [2]. The layers were arranged in a $[0 / 45 /-45 / 90]_{n s}$ stacking sequence, where $n$ was 2 for nominal thickness of $6.35 \mathrm{~mm}$ and 8 for nominal thickness of $25.4 \mathrm{~mm}$. The fibers themselves consisted of yarns with a yield of 1.12 $\mathrm{m} / \mathrm{g}$ and a spacing of 4.53 yarns $/ \mathrm{cm}$. Given the composite, fiber, and matrix densities of $1.57,1.78$, and $1.25 \mathrm{~g} / \mathrm{cm}^{3}$, respectively, and assuming that the void

Corresponding author: $\underline{\text { schocron@swri.edu }}$ 
content of the composite is negligible, the approximate fiber and matrix volume fractions within the composite are $60 \%$ and $40 \%$, respectively.

AEC also provided a 0.93 in-thick $(23.4 \mathrm{~mm})$ neat resin plate to extract resin-only specimens. The resin material was the same than the one used in the 2-D and 3-D composites (CYCOM PR 520). The resin was tested in torsion, tension, "delamination", and compression at low, intermediate, and high strain-rates.

\subsection{Specimens Machined and Test Matrices}

\subsubsection{Neat Resin Specimens}

The neat resin specimens were machined to obtain three different specimen geometries: tension, torsion, and delamination. The tensile specimens were flat with a nominal thickness of $1 / 4$ in $(6.35 \mathrm{~mm})$, gage length 2.5 in $(63.5 \mathrm{~mm})$, and gage width $0.5 \mathrm{in}(12.7 \mathrm{~mm})$. The specimens flared out at the grip area to ensure failure in the gage section. The torsion specimens were machined as cubes with a side length of $.93 \mathrm{in}(23.4 \mathrm{~mm})$ and a notch with a gage length of 0.12 in $(3.05 \mathrm{~mm})$ and a radius of 0.06 in $(1.5 \mathrm{~mm})$. The delamination specimens were hourglass-shaped with a square base of 1.38 inch $(35.05 \mathrm{~mm})$, a height of $0.93 \mathrm{in}(23.4 \mathrm{~mm})$, a gage section width of .75 in $(19.05 \mathrm{~mm})$, and gage section radius of 0.06 in $(1.5 \mathrm{~mm})$. The compression cylinders were machined as right circular cylinders with a 0.45 -in $(11.43 \mathrm{~mm})$ diameter, and a length of $0.91 \mathrm{in}$ $(23.11 \mathrm{~mm})$.

Table 1. Neat Resin Specimens Tested.

\begin{tabular}{ccc}
\hline Specimen & $\begin{array}{c}\text { Specimen } \\
\text { ID }\end{array}$ & $\begin{array}{c}\text { Strain Rate } \\
\left(\mathbf{s}^{\mathbf{- 1}}\right)\end{array}$ \\
\hline Tensile & MP1-Tn & $10^{-3}$ \\
Torsion & MR-nZ & $10^{-3}$ \\
Delamination & MD-n & $10^{-3}, 1$ \\
Compression & Compn & $10^{-3}, 1,10^{3}$ \\
\hline
\end{tabular}

Table 1 provides the ID for each of the specimens and the number of specimens tested. As seen in the table, tensile and torsion tests were only performed at quasistatic strain rates. The delamination tests were performed also at intermediate rates and the compression tests at intermediate and high strain-rates.

\subsubsection{Two-dimensional CFRP Specimens}

Table 2 shows the 2-D composite specimens that were tested. The large rectangular tensile specimens had a length of $10 \mathrm{in}(254 \mathrm{~mm})$. (gage length $4 \mathrm{in} . / 10.16 \mathrm{~mm})$, width of 1 in. $(25.4 \mathrm{~mm})$, and a thickness of $1 / 4$ in $(6.35 \mathrm{~mm})$. These were used to measure the modulus and strength of the composite and allowed for validation of the mesoscale geometry used in computations. Specimens were extracted in the longitudinal $\left(0^{\circ}\right)$ and transverse direction $\left(90^{\circ}\right)$ to check for any anisotropy.
Small specimens were also tested in tension because the Hopkinson bar apparatus does not allow testing large tensile specimens. The width and thickness of the small specimens is only $1 / 4$ in and $1 / 8$ in respectively, with a gage length of 0.3 inches. The thickness was reduced by machining down on both sides of the original plate to obtain a symmetric specimen. The final layup for the specimens was $[90 /-45 / 45 / 0]_{\mathrm{s}}$ for the $T$ specimens and $[0 / 45 /-45 / 90]_{\mathrm{s}}$ for the $L$ specimens, where $0^{\circ}$ is the direction of the load. The small specimens tested at quasistatic rates provided a "size effect" when compared to the large specimens. The rate effect was found by comparing the high strain-rate tests on small specimens with the quasistatic.

The delamination and torsion specimens for the 2-D composite were similar to the ones described for the neat resin. Note that the torsion specimens with yarns going through the gage section (2R-nX and 2R-nY) used a smaller gage section because the torque needed to fail the large specimens was larger than the limit of the torsion machine.

Table 2. 2-D Specimens Tested.

\begin{tabular}{ccc}
\hline Specimen & $\begin{array}{c}\text { Specimen } \\
\text { ID }\end{array}$ & $\begin{array}{c}\text { Strain } \\
\text { Rate }\left(\mathbf{s}^{-1}\right)\end{array}$ \\
\hline Tensile, small, longit. & $2 \mathrm{LS}-\mathrm{n}$ & $10^{-3}, 10^{3}$ \\
Tensile, small, transverse & $2 \mathrm{TS}-\mathrm{n}$ & $10^{-3}, 10^{3}$ \\
Tensile, large, transverse & $2 \mathrm{~T}-\mathrm{n}$ & $10^{-3}, 1$ \\
Tensile, large, longitudinal & $2 \mathrm{~L}-\mathrm{n}$ & $10^{-3}, 1$ \\
Torsion, $\mathrm{x}$ - direction & $2 \mathrm{R}-\mathrm{nX}$ & $10^{-3}$ \\
Torsion, y - direction & $2 \mathrm{R}-\mathrm{nY}$ & $10^{-3}$ \\
Torsion, $\mathrm{z}$ - direction & $2 \mathrm{R}-\mathrm{nZ}$ & $10^{-3}$ \\
Delamination & $2 \mathrm{D}-\mathrm{n}$ & $10^{-3}, 1$ \\
\hline
\end{tabular}

\subsection{Specimens for Ballistic Tests}

Square composite panels were manufactured by AEC with thicknesses of $1 / 4$ in $(6.35 \mathrm{~mm})$ and 1 in $(25.4 \mathrm{~mm})$ for ballistic tests. The 2-D unidirectional panels were identical to the ones used for the mechanical characterization experiments: IM7 carbon fibers bonded with PR520 high toughness epoxy matrix. This was a purposeful decision, as it is expected that the material tests will provide the constants to be used in the ballistic computations without further need to calibrate. I.e. that the ballistic tests are intended to be a validation of the constants obtained with the mechanical laboratory tests.

Table 3 summarizes the ballistic panels tested and provides the thickness of the panels $(\mathrm{T})$ and the typical areal density (Ad) measured in $\mathrm{kg} / \mathrm{m}^{2}$ and $\mathrm{lb} / \mathrm{ft}^{2}$.

Table 3. Ballistic Specimens, Thickness, and Areal Density.

\begin{tabular}{|c|c|c|}
\hline Specimen & T (in) & $\begin{array}{c}\operatorname{Ad~} \mathbf{k g} / \mathrm{m}^{2} \\
\left(\mathrm{lb} / \mathbf{f t}^{2}\right)\end{array}$ \\
\hline 2-D IM7/PR520 & 1 & $3.9(8.0)$ \\
\hline 2-D IM7/PR520 & 0.25 & $0.97(2.0)$ \\
\hline
\end{tabular}


In addition to the materials mentioned above, SwRI also received IM7 carbon fiber yarns and a single layer of woven carbon fiber yarns. These were used to test single yarns under ballistic impact and helped assessing the yarn impact theory.

\section{METHODS}

\subsection{Quasistatic Tensile and Compression Tests}

Aluminum tabs $1 / 8$-inch-thick $(3.17 \mathrm{~mm})$ were glued on the ends with high shear strength epoxy glue. Four specimens $(2 \mathrm{~T}-2,2 \mathrm{~L}-10,3 \mathrm{~T}-2$, and $3 \mathrm{~L}-10)$ were strain gaged with two gages in the front and two gages on the side. This allowed the determination of the in-plane and out-of-plane Poisson's ratio for the composite.

All the tests included a clip-gage so that the strain measured could be compared with the one recorded from the strain gages. Hydraulic grips were used to secure proper gripping. Approximate nominal strain rates of $10^{-4} \mathrm{~s}^{-1}$ and $10^{-1} \mathrm{~s}^{-1}$ were achieved during the tests. The frame used for the test was an MTS with a load cell of 100 kip $(445 \mathrm{kN})$. Neat resin specimens did not have strain gages glued but the strain was recorded using a clip gage.

Quasistatic tensile tests were also performed on small composite specimens. The objective of these tests is mainly to explore the size effect when testing large vs. small specimens. Only small specimens can be tested at high strain-rates in the Hopkinson bar so it is important to determine if the difference in the test results is due to the geometry or the strain rate.

The grips are a major concern when using the Hopkinson bar as they can interfere with the signals. Building on previous experience, it was decided that slot grips may provide more reliable results. The specimen is glued to the grip with the same high shear strength glue used for the tabs of the large specimens. Note that the clip gage does not fit in the specimen gage length, which was designed with the Hopkinson bar test in mind. This means that the strain measurement is not reliable unless corrected for compliance. Two of the specimens (2TS-09 and 2TS-10) were strain gaged to provide the compliance correction.

The compression tests were performed in the same MTS machine as the small tensile tests. Only neat resin specimens were used for compression. The clip gage was placed on top and bottom platens. The compliance of the resin is much larger than the compliance of the platens so compliance correction was not needed for this test. All the compression tests use molybdenum grease to reduce the friction between the platen and the specimen.

\subsection{Hopkinson Bar Tensile and Compression Tests}

The one-inch diameter direct-tensile Hopkinson bar was used for the high strain rate tests, see Lindholm [3] for the assumptions and details of principles for this apparatus.

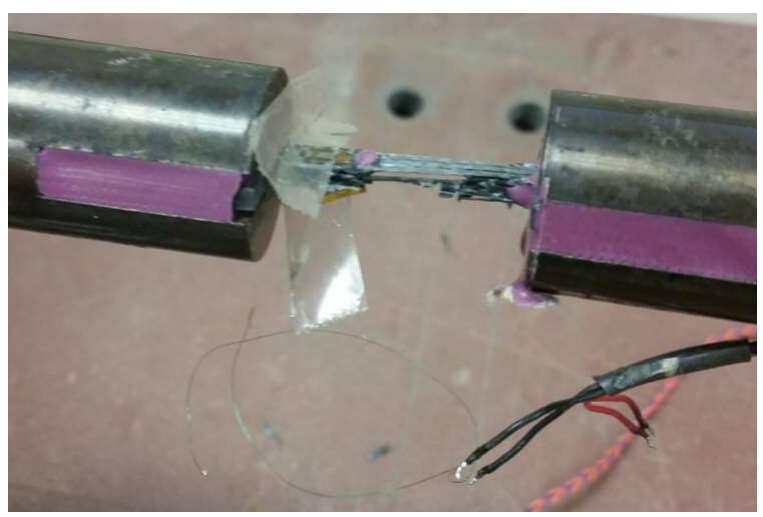

Fig. 1. Photograph of a CFRP specimen after being tested in the bar.

The input and output bars were made of Vascomax C350 maraging steel. The length of each of the bars is 10 feet. The 48-inch projectile provided a 500- $\mu$ s-long pulse, which failed the specimen in all the tests in the first wave pass. Strain gages were glued on both sides of the composite specimens tested in the SHPB. The slot grips were used as they interfere minimally with the input and output waves.

\subsection{Ballistic Tests}

Two types of ballistic tests were performed under this program: 1) carbon fiber single-yarn ballistic impacts, and 2) ballistic tests of laminates. The objective of the single-yarn ballistic impact was to assess if wave propagation in carbon fiber follows the classical theory. The ballistic tests on laminates were used as validation of the computer material model developed for this program.

The 0.30 cal fragment simulator projectile was used for testing all the laminates and approximately half the impacts on carbon fiber yarns. A steel right circular cylinder (1/4 in diameter and 1.25 in long) was used for the other half of the yarn impacts.

The target laminates were simply supported by two steel beams ( 7 in. apart) with 4 losely tightened c-clamps on the four corners of the plate. A mirror was placed behind the target so that the two Phantom v7.11 cameras could record the motion of the back of the target. A pattern was sprayed with white paint on the back of some of the targets to perform digital image correlation after the test and obtain the displacement history of the back surface.

Additionally, in all the tests, a third camera (Shimadzu HPV-X2) recorded the impact event from the lateral side of the target. This camera is much faster than the Phantoms and can provide submicrosecond pictures of the evolution of the impact pyramid (apex and base width), which is used as a validation for the numerical models. More details on the ballistic tests can be found in [4]

In summary, the relevant information from the laminate ballistic tests was: a) impact velocity, b) residual velocity, c) ballistic limit for each of the laminates of interest, and d) displacement history of the back of the target. 


\section{TEST RESULTS}

\subsection{Tests on the PR520 Matrix Material}

\subsubsection{Tensile Tests on Resin}

The tensile tests results on resin only material are reported in Figure 2. Maximum tensile strength is approximately $80 \mathrm{MPa}$. The first test MP1-T1 should be considered invalid as the grips cracked the specimen. The other tests provide strains to failure between 5 and $12 \%$. The strains were measured with a clip gage. Due to the brittle nature of the matrix it is not surprising to see a large scatter in the results.

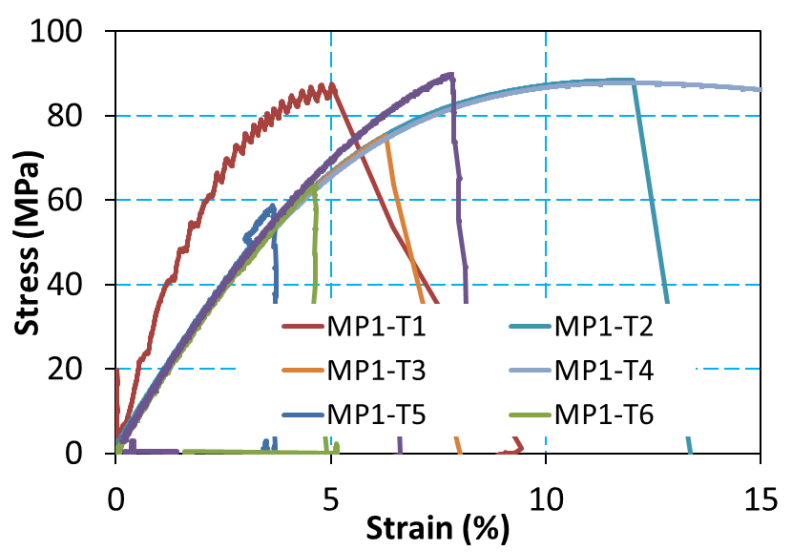

Fig. 2. Neat resin tensile test results.

\subsubsection{Compression Tests}

Compression tests on neat resin were performed at quasistatic and intermediate rates in the MTS machine and at high strain rates in the Hopkinson bar. All the specimens were the same geometry: diameter 0.45 in and length 0.911 in Figure 3compares the tests for the three strain-rates of interest. Note that the specimen is stronger at higher strain-rates. Engineering failure strains for the quasistatic and intermediate rates are, typically, $60 \%$ and $50 \%$ respectively. Strength increases significantly at very high rates of $10^{3} \mathrm{~s}^{-1}$ (red curves). Note that the end of the red curves is not because of the failure of the specimen but just because the pulse from the projectile ends prematurely.

\subsection{Tensile Tests on Laminates}

\subsubsection{Tensile Tests on Large Specimens}

The large tensile specimens provide very relevant information like the elastic modulus and the strength of the laminate. This information allows checking the mesoscale model as well as seeing if the rule of mixtures applies for this composite. Other relevant information is the Poisson's ratio in different directions. The specimens are large enough to include many yarns in the width direction so that the specimen is representative of the real material. For example for the 2-D composite the width of the specimen includes 10 yarns.

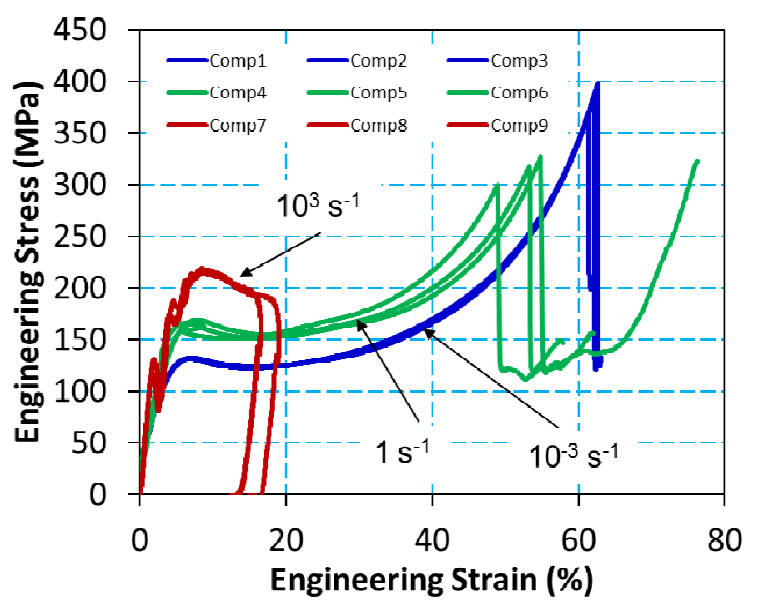

Fig. 3. Compression tests performed on neat resin at quasistatic, intermediate, and high strain-rates.

Gripping of composite specimens can be an issue as the specimen may delaminate instead of fail in tension. For some composite laminates, like the ones based on ultra-high-molecular-weight polyethylene, the in-plane shear strength is very low and, consequently, the shear load applied by the grips cannot be properly transmitted to the center of the specimen. Hence, the specimen does not fail in tension but is just delaminated in shear. This does not seem to be happening with the large CFRP specimens tested in this project but it is likely that the small specimens are suffering from this issue.

Figure 4 shows the results from the tensile tests of the large 2-D specimens. $L$ and $T$ are for longitudinal and transverse specimens. Thick lines are for quasistatic tests performed at $10^{-4} \mathrm{~s}^{-1}$ while thin lines are for tests performed at $0.7 \mathrm{~s}^{-1}$ (the fastest travel on the MTS machine).

The elastic modulus is the same for both directions, implying that the specimen properties are the same but it is important to notice that the longitudinal specimens seem to be failing systematically earlier than the transverse. This is likely an artifact from gripping. The direction of the tensile load is the same than that of the yarns on the first layer for the $L$ specimens. This layer is probably the one showing up as the first "failure-step" in the stress-strain curve. It does not mean that it is the first layer to fail as the 45 and $90 \mathrm{deg}$. layers can probably carry only a small load so may be failing without noticeable change in load carrying capability.

\subsubsection{Tensile Tests on Small Specimens}

Figure 5 shows the tensile tests for the small specimens. Only two of the tests (2TS-9 and 10) were strain-gaged. All the other tests were corrected for compliance to match the modulus of these two tests. Figure 6 compares the large (2T-2) and small (2TS) specimens. The small specimens have the same modulus as the large 
specimens, although there is some scatter in the data and only two tests were performed with strain gages.

Interestingly, the apparent strength of the small specimens is much smaller, i.e. $\sim 500 \mathrm{MPa}$ instead of the 700-800 $\mathrm{MPa}$ for the larger specimens. The reason is, again, likely the gripping. The specimens are mostly delaminating and not failing in tension. So the small specimens do not provide accurate strength information.

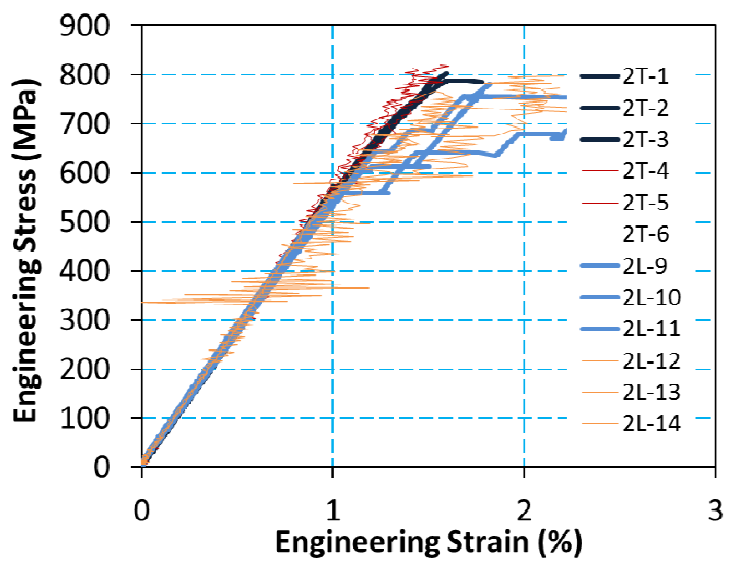

Fig. 4. Tensile tests on large unidirectional specimens. Thick lines are for quasistatic tests while thin lines for intermediate strain rate tests.

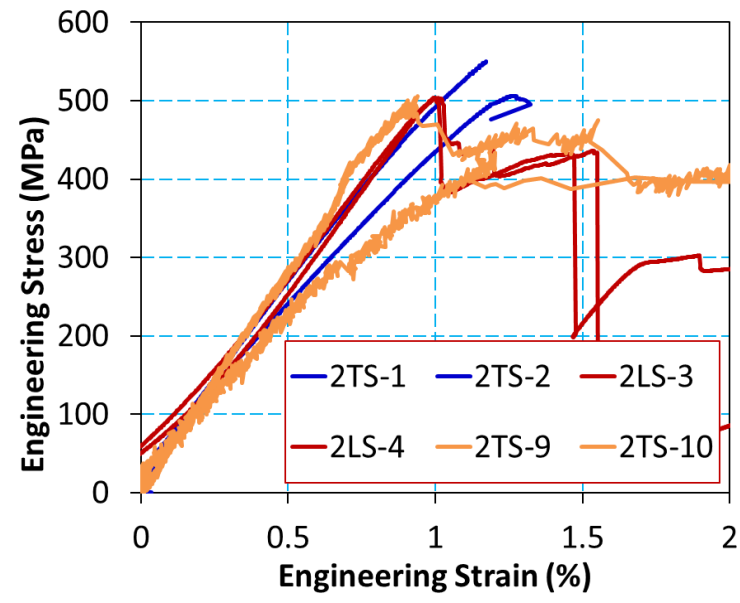

Fig. 5. Quasistatic tests performed on small tensile specimens.

\subsubsection{Hopkinson Bar Tensile Tests}

Four small tensile specimens, similar to the ones discussed in the previous section, were tested in the Hopkinson bar. All these specimens were strain gaged but the gages failed very early during the tests, at a little over $1 \%$ strain, but confirmed that the strain provided by integrating the reflected signal of the input bar is correct.

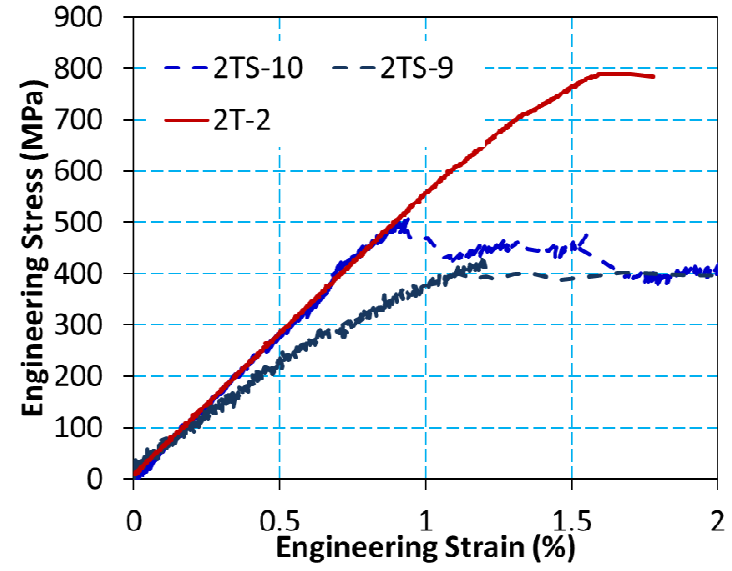

Fig. 6. Comparison of small and large specimens.

Figure 7 shows the results for four Hopkinson bar tests. Except for 2LS-6, the tests provide very similar strength data to the quasistatic tests (between 400 and $500 \mathrm{MPa}$ ). The higher strength in test 2LS-6 is speculated to happen due to that particular specimen gripping better than the others.

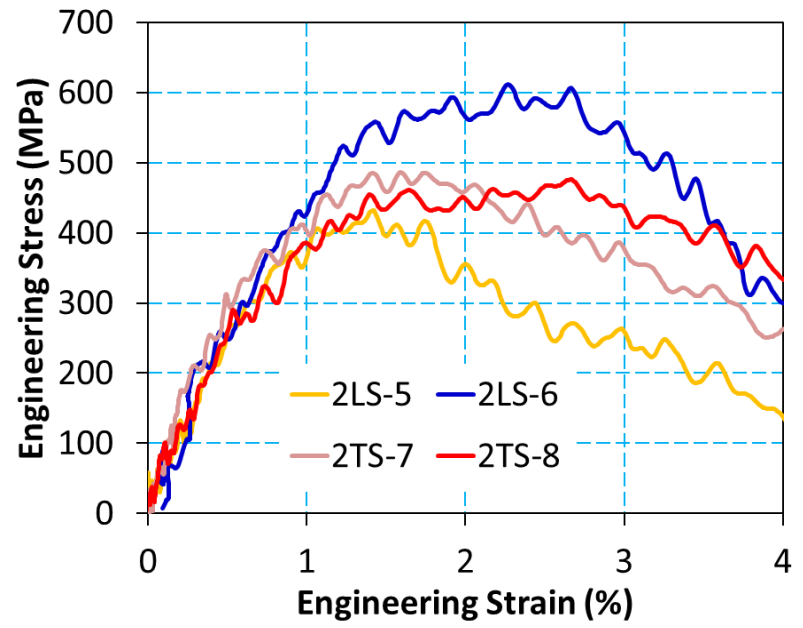

Fig. 7. Hopkinson bar tests on small tensile specimens. Results for all the tests performed.

In summary, the above tests seem to indicate that there is not a size effect (other than the gripping issue) between large and SHPB samples. It is difficult to reach a conclusion as far as strain-rate effects in this material because the response is dominated by grip effects. It will be shown in the simulation section that not considering any strain-rate effect allows for prediction of the ballistic limit of the laminates. This strongly suggests that the strain-rate effects are not important for these laminates.

\subsection{Ballistics Results}

Due to lack of space the ballistics results are presented together with the computations in the next section. 


\section{MESOSCALE COMPUTER MODEL}

\subsection{Unidirectional Composite Architecture}

The architecture for the finite element model followed closely the geometry of the real material: same fiber and matrix content, yarn size and count, etc. The matrix elements are constant stress elements, which contain only one integration point. However, the yarn elements are fully integrated elements containing 8 integration points. Unlike constant stress elements, fully integrated elements can resolve bending stresses, which are thought to be an important aspect of the carbon fiber yarns' deformation and failure response. In fact, single yarn impact tests and computations showed that to match the experimental critical velocity ${ }^{a}$ fully integrated elements needed to be used. Because of the 45/-45 degree layers in the composite, adjacent layers within a specimen mesh cannot share nodes. Therefore, interaction between mesh layers is handled using a tiebreak contact condition in LS-DYNA. The stresses required to break the ties are the same as for the fiber/yarn interface model.

\subsection{Simulation of Material Tests}

Simulations were performed to reproduce the tensile, delamination, and torsion tests. The prediction from the model was, in general, excellent, matching the tensile (see Figure 8), delamination, and torsion in the $\mathrm{z}$ direction very closely. The only discrepancy was seen in the failure of the $\mathrm{x}$-direction torsion tests, although the first part of the curve up to the maximum torque is properly captured. This is a very difficult test to compute because the yarns cross the gage section and are torqued along their length.

\subsection{Simulation of Ballistic Tests}

The same material architecture was used for both the material and ballistic test simulations. Ballistic limits and residual velocities were properly captured for the two laminates thicknesses, see Figure 9. The deflection history recorded with the ultra-high-speed camera also compared very well with the simulations.

\section{CONCLUSIONS}

The methodology followed to obtain a predictive mesoscale model for ballistic impact was successful. This method consists of tensile, compression, torsion, and delamination tests of the neat resin and the laminate at various strain rates. Highly instrumented ballistic tests were also performed. The result was a predictive model that accurately captures the behavior for material and ballistic tests (ballistic limits, deflection, etc.).

\footnotetext{
${ }^{\text {a }}$ Impact speed that immediately produces the failure of the yarn.
}

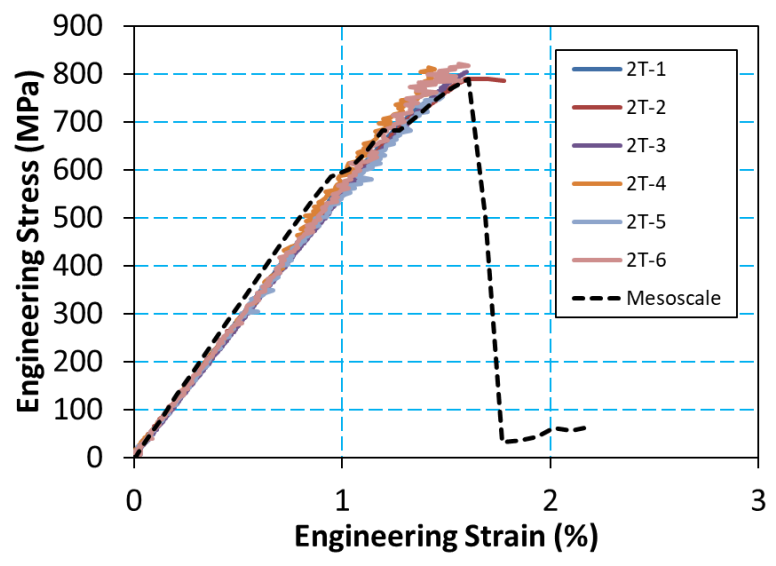

Fig. 8. Simulated engineering stress-strain curve compared to tensile test data from the 2D CFRP composite.

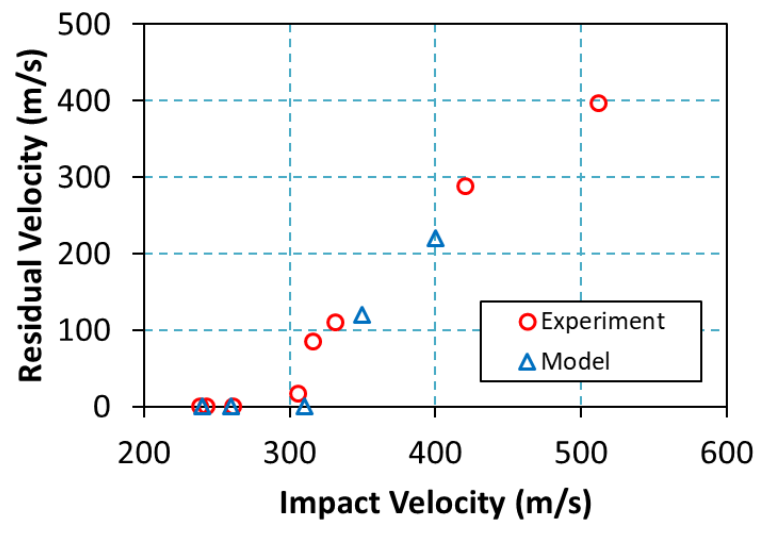

Fig. 9. Residual velocity is plotted against impact velocity for the 6.35-mm-thick 2D CFRP panels.

\section{References}

1. Hexcel Corporation, "HexTow IM7 (5000) Product Data" (2007)

2. Cytec Engineered Materials, "Cycom PR 520 RTM Resin System" (2012)

3. U. S. Lindholm, "Some Experiments with the SplitHopkinson Pressure Bar," J. Mech. Phys. Solids, vol. 12, p. 317 (1964)

4. K. Warren, H. Bayraktar, L. Warner, S. Chocron, and R. Bigger, "Ballistic Impact Performance of Threedimensional Woven Composites," in Proceedings of the SAMPE Conference, Seattle, USA, May 2017 (2017) 